\title{
Absolute structure and absolute configuration
}

\author{
H. D. Flack* AND G. Bernardinelli \\ Laboratoire de Cristallographie, University of Geneva, 24 quai Ernest Ansermet, CH-1211 Genève 4, Switzerland. \\ E-mail: howard.flack@cryst.unige.ch
}

(Received 13 January 1999; accepted 19 March 1999)

\begin{abstract}
Fundamental notions concerning absolute structure and absolute configuration, and their determination from single-crystal diffraction measurements, are presented and reviewed. A glossary of terms with definitions useful in this field is provided. For absolute structure and its determination, the separate but interacting influences of the structure and the inversion-distinguishing power of an X-ray diffraction experiment with dispersive scatterers are examined. Important experimental and algorithmic details of the current methods used for absolute-structure determination are provided. Characterization of crystals for absolute-structure determination and of molecules for absolute-configuration determination are treated. Attention is given to the analysis of absolute structure and absolute configuration in twinned crystals.
\end{abstract}

\section{Glossary of terms}

Absolute configuration. The spatial arrangement of the atoms of a physically identified chiral molecular entity (or group) and its stereochemical description (e.g. $R$ or $S, P$ or $M, D$ or $L$ etc.).

Absolute structure. The spatial arrangement of the atoms of a physically identified noncentrosymmetric crystal and its description by way of unit-cell dimensions, space group and representative coordinates of all atoms.

Chiral. Having the property of chirality (Moss, 1996).

Chirality. The geometric property of a rigid object (or spatial arrangement of points or atoms) of being nonsuperposable on its mirror image; such an object has no symmetry operations of the second kind (a mirror plane, $m$; a centre of inversion, $\overline{1}$; a roto-inversion axis, $\bar{N})$. If the object is superposable on its mirror image, the object is described as being achiral [Moss (1996), modified for H-M symbols].

Chirality sense. The property that distinguishes enantiomorphs. The specification of two enantiomorphic forms by reference to an oriented space, e.g. of a screw, a right-threaded one or a left-threaded one. The expression opposite chirality is short for opposite chirality sense (Moss, 1996).

Enantiomer. One of a pair of molecular entities which are mirror images of each other and nonsuperposable (Moss, 1996).
Enantiomerically pure/enantiopure. A sample in which all molecules have (within the limits of detection) the same chirality sense. Use of homochiral as a synonym is strongly discouraged (Moss, 1996).

Enantiomorph. One of a pair of chiral objects or models that are nonsuperposable mirror images of each other (Moss, 1996).

Flack (1983) parameter. The parameter $x$ in the structure-amplitude equation

$$
G^{2}(h, k, l, x)=(1-x)|F(h, k, l)|^{2}+x|F(\bar{h}, \bar{k}, \bar{l})|^{2}
$$

(Flack, 1983).

Inversion twin. An inversion twin consists of centrosymmetrically related crystalline domains. The symmetry operation relating domain structures in an inversion twin is that of a centre of symmetry.

Racemate. An equimolar mixture of a pair of enantiomers. It does not exhibit optical activity. The chemical name or formula of a racemate is distinguished from those of the enantiomers by the prefix $( \pm)$ or rac- (or racem-) or by the symbols $R S$ and $S R$ (Moss, 1996).

Racemic. Pertaining to a racemate (Moss, 1996).

Racemic compound. A crystalline racemate in which the two enantiomers are present in equal amounts in a well defined arrangement within the lattice of a homogeneous crystalline addition compound (Moss, 1996).

Racemic conglomerate. An equimolar mechanical mixture of crystals, each one of which contains only one of the two enantiomers present in a racemate. The process of its formation on crystallization of a racemate is called spontaneous resolution, since pure or nearly pure enantiomers can often be obtained from the conglomerate by sorting (Moss, 1996).

\section{Introduction}

... la dissolution laisse déposer, après quelques jours, des cristaux qui ont tous exactement les mêmes angles, le même aspect; et pourtant, à coup sûr, l'arrangement moléculaire dans les uns et les autres est tout à fait différent. (Pasteur, 1848.)

Absolute structure is a crystallographer's term and applies to noncentrosymmetric crystal structures. Absolute configuration is a chemist's term and refers to 
chiral molecules. Note particularly that both the entity under consideration, viz crystal structure versus molecule, and the symmetry restrictions, viz noncentrosymmetric versus lack of mirrors, centres of symmetry and roto-inversions, are different. Both terms concern the complete specification of the spatial arrangement of atoms with respect to inversion. Discussion and justification of our choice of definition of these two terms will be deferred until a later section. As the word absolute appears as a qualifier, an impression is generated that a complete, justified, invariant and transferable specification of the spatial arrangement of the atoms is being made available. In practice, such desirable conditions may not have been achieved.

Structure analysis and information technology have advanced greatly since other authors (Glazer \& Stadnicka, 1989; Jones, 1984a,b, 1986b; Rogers, 1975, 1981) have warned about the misuse and misunderstanding of the terms absolute structure and absolute configuration and related concepts. The advent of the Crystallographic Information File, CIF (Hall et al., 1991), has created the requirement for the clear definition of data items concerned with the reporting of absolute structure and absolute configuration and permits their machine validation. Modern single-crystal structure-analysis systems use the Flack parameter (Flack, 1983; Bernardinelli \& Flack, 1985, 1987) to estimate absolute structure, a method which was in its infancy at the time of the previous reports. Moreover, the availability of area detectors and synchrotron-radiation sources offers improved opportunities for data collection, which have made the determination of absolute structure more widely applicable than previously.

The current paper presents the fundamental notions associated with the determination and reporting of absolute structure and absolute configuration from crystal structure analysis. The topics treated have been chosen on the basis of private communications with the authors and of a comprehensive study of all papers in Acta Crystallographica Section C, Vol. 52 (1996). The latter will be presented in detail in a future paper (Flack \& Bernardinelli, 1999). The overall objective is to identify shortcomings in present practice and to provide a basis for improvement. To this effect, we provide a glossary of important terms in this field, have negotiated new or improved definitions for CIF data names and, with the further paper, provide a checklist in algorithmic form for use by publishers of crystal-structure journals.

The terms in the glossary $(\S 1)$ related to absolute structure and absolute configuration have been gathered together with the needs of the principal user groups in mind, viz structure analysts and chemists. The terms chosen for inclusion are those that have been used and adhered to in the present article and the definitions are thought to be the clearest available corresponding to current usage. Their source has been cited if not original to the present paper. Of particular interest was Basic
Terminology of Stereochemistry, IUPAC Recommendations 1996 (Moss, 1996). No discussion is presented of terms not included in the list.

\section{Absolute structure}

\subsection{Inversion-distinguishing power}

The central problem in absolute-structure determination is the capacity to distinguish between an image of the crystal structure and that of a centrosymmetrically related one. A successful structure analysis on a crystal with a noncentrosymmetric structure will always produce a list of atomic coordinates corresponding to a single noncentrosymmetric spatial arrangement of atoms in the crystal, whether or not the data are capable of distinguishing between this arrangement and its inverse. Where the inversion-distinguishing power is low, the result is in reality a choice between two centrosymmetrically related images, although only one image of the structure is presented. One may say that the structure determination is ambiguous. On the other hand, where the inversion-distinguishing power is sufficiently high, one may assert that the model and the crystal as mounted on the diffractometer correspond one to another. The structure determination is potentially absolute.

\subsection{Right-handed axes}

As emphasized and discussed by Rogers (1975), right-handed sets of axes must be used at every stage of an analysis of absolute structure. Of particular danger for the structure analyst are basis transformations performed to bring the unit cell into a standard setting.

\subsection{Inversion twins}

Crystals may be twinned by growth or phase transformation. Of relevance to the study of absolute structure and absolute configuration is the occurrence of inversion twins. Twinning by inversion is characteristic of crystal structures displaying a noncentrosymmetric space group. In an inversion twin, the crystal lattice (i.e. the lattice translations after removing the atoms) is maintained throughout the whole volume of the sample, but the atoms and molecules take up either one spatial arrangement or the inverted one depending on the position within the crystal. A visual model of an inversion twin, applicable to chiral crystal structures, is to imagine the individual components of a racemic conglomerate being stuck together with their lattices being perfectly oriented. The inversion-twinned crystal is an oriented solid-state mixture of inverted structures. Inversion-twinned crystals do not form from an enantiopure sample of a substance. 


\section{Determination of absolute structure by $X$-ray diffraction with dispersive scatterers}

We owe to Coster et al. (1930) the first demonstration that X-ray diffraction using anomalous scattering can distinguish a noncentrosymmetric crystal structure from its inverted image. Previous to this experiment, the inversion-distinguishing power of X-ray diffraction was considered to be zero as expressed in Friedel's law. X-ray anomalous scattering is a second-order effect, making its inversion-distinguishing power small but nevertheless significant. By comparison, optical systems using visible light and lenses have a very high inversion-distinguishing power. The presence of significant dispersive scatterers in a noncentrosymmetric crystal structure leads to the intensity distribution in the X-ray diffraction pattern revealing the true point symmetry of the structure (crystal class), rather than that of its supergroup containing a centre of symmetry (Laue symmetry). Modern technology is such that the observation of intensity differences between Friedel opposites $(h k l$ and $\bar{h} \bar{k} \bar{l})$ is now the rule rather than the exception. It follows that, with the capacity to produce data displaying the symmetry of the crystal class, it is now routinely possible to distinguish a noncentrosymmetric structure from its inverse, thus opening the way to the determination of absolute structure by X-ray diffraction from a single crystal.

An advantage of distinguishing a noncentrosymmetric structure from its inverse in the refinement of a crystal structure is the more realistic modelling that it affords, with the consequent improvement in statistical measures of fit. The capacity of a refinement to adapt to an inverted model can be startling for a crystal structure in a space group in which the origin cannot be fixed with respect to the symmetry elements of the space group (point groups: 1, 2, m, mm2, 4, 4mm, 3,3m, $6,6 \mathrm{~mm})$. Under the conditions leading to a polar dispersion error (Ueki et al., 1966; Cruickshank \& McDonald, 1967), displacement of the apparent position of the atoms along the free directions will compensate for any mistake in the absolute structure or anomalous-dispersion contribution. Thus the need to avoid systematic errors, which may arise in the atomic positional coordinates and bond lengths when a polar dispersion error occurs, turns realistic modelling of the inversion possibilities from an advantage into a necessity. It is also well established (Jones et al., 1988) that an inverted structure model gives rise to false, although plausible, ghost atoms in an electron-density map.

The main routine method for distinguishing a noncentrosymmetric structure from its inverse when anomalous scattering is significant is use of the Flack (1983) parameter. The most common alternative to this is Hamilton's (1965) $R$-factor-ratio test.

\subsection{Flack (1983) parameter}

In essence, any noncentrosymmetric crystal is treated as being a twin by inversion and the fractional contributions of the twin components are considered as variable during the least-squares refinement of the crystal structure. The underlying squared structure-amplitude equation is

$$
G^{2}(h, k, l, x)=(1-x)|F(h, k, l)|^{2}+x|F(\bar{h}, \bar{k}, \bar{l})|^{2} .
$$

When $x$ takes a value of zero, the atomic arrangement of the model and the crystal are identical; when $x$ takes a value of one, the atomic arrangement of the model and that of the crystal are inverted one with respect to the other. For a twinned crystal, $x$ gives a measure of the relative amounts of the structure and its inverse in the crystal. Full details of the use and advantages of this approach have been given by Flack (1983), Bernardinelli \& Flack $(1985,1987)$ and Flack \& Schwarzenbach (1988). The reciprocal of the standard uncertainty of $x$ is a direct measure of the inversion-distinguishing power of the diffraction measurement, while $x$ itself is a way of indicating what has been distinguished. Under conditions where a polar dispersion error may occur, one witnesses large correlations between the Flack (1983) parameter and the atomic coordinates along the originfree directions.

The implementation of the Flack parameter in the widely used refinement program SHELXL93/ SHELXL97 (Sheldrick, 1993/1997) uses a special algorithm requiring care in its interpretation. Details will be given by Flack \& Bernardinelli (1999).

\subsection{Hamilton's (1965) R-factor-ratio test}

As applied to absolute-structure determination, Hamilton's (1965) test was subject to a very critical analysis by Rogers (1981), casting doubt on its fundamental validity in this case. This work gave rise to a flurry of activity (Jones, 1984a,b, 1986a,b; Jones \& Meyer-Bäse, 1987; Flack, 1983; Bernardinelli \& Flack, 1985, 1987; Glazer \& Stadnicka, 1989) and the emergence of the Flack (1983) parameter. The basis of Hamilton's method is the comparison of the conventional or weighted $R$ factors from two models of the structure, i.e. the refined model and its inverse. The study by Flack \& Bernardinelli (1999) of published structures shows that Hamilton's test is almost always being applied incorrectly in current practice. It is worth mentioning yet again (Flack, 1983; Bernardinelli \& Flack, 1985) that the Hamilton test cannot take account of the effects of inversion twinning. $R$ values that are judged to be insignificantly different by Hamilton's test may represent situations where either the inversiondistinguishing power is too low or where the crystal is twinned by inversion. 


\subsection{Enhancing inversion-distinguishing power}

It is most instructive to examine the conditions controlling the inversion-distinguishing power in an $\mathrm{X}$-ray diffraction experiment with dispersive scatterers. This will be studied by a simple model. Let the nondispersive light atoms make a contribution $\mathbf{F}_{L}(\mathbf{h})$ to the structure factor $\mathbf{F}(\mathbf{h})$ of

$$
\mathbf{F}_{L}(\mathbf{h})=L\left[\cos \left(\varphi_{L}\right)+i \sin \left(\varphi_{L}\right)\right]
$$

obtained by summing over all light atoms in the unit cell. Let the dispersive heavy atoms be all of the same kind and let their contribution $\mathbf{F}_{H}(\mathbf{h})$ (obtained by summation over all heavy atoms in the unit cell) to $\mathbf{F}(\mathbf{h})$ be

$$
\mathbf{F}_{H}(\mathbf{h})=\left(H+i H^{\prime \prime}\right)\left[\cos \left(\varphi_{H}\right)+i \sin \left(\varphi_{H}\right)\right] .
$$

Then

$$
\mathbf{F}(\mathbf{h})=\mathbf{F}_{L}(\mathbf{h})+\mathbf{F}_{H}(\mathbf{h})
$$

and the difference in intensity between Friedel opposites

$$
\Delta(\mathbf{h})=|\mathbf{F}(\mathbf{h})|^{2}-|\mathbf{F}(\overline{\mathbf{h}})|^{2}=4 L H^{\prime \prime} \sin \left(\varphi_{L}-\varphi_{H}\right) .
$$

The inversion-distinguishing power depends on the values of $\Delta$ for all reflection pairs in a data set and a rough measure is obtained from

$$
\langle|\Delta|\rangle \simeq 4\langle L\rangle\left\langle\left|H^{\prime \prime}\right|\right\rangle\left\langle\left|\sin \left(\varphi_{L}-\varphi_{H}\right)\right|\right\rangle .
$$

$\langle|\Delta|\rangle$ will increase as the values of $\Delta$ increase and it will be zero when $\Delta$ is systematically zero over all reflections. This occurs when $(a) L=0$, i.e. all atoms in the unit cell are dispersive but of the same kind as occurs in the structure of a chemical element even if noncentrosymmetric, $(b) H^{\prime \prime}=0$, i.e. there are no dispersive scatterers as occurs in the application of Friedel's law, and $(c) \sin \left(\varphi_{L}-\varphi_{H}\right)=0$ as occurs in centrosymmetric structures regardless of the choice of origin. The conditions that lead to a large value of $\langle|\Delta|\rangle$ are evident. Both $\langle L\rangle$ and $\left\langle H^{\prime \prime}\right\rangle$ must be as large as possible, i.e. there must be sizeable nondispersive and dispersive contributions in the unit cell. $\left\langle H^{\prime \prime}\right\rangle$ may be increased by a change of X-ray wavelength. In general, the trigonometric term in $\langle|\Delta|\rangle$ will take a nonzero value but it will tend to zero if $\varphi_{L}$ and $\varphi_{H}$ are strongly correlated, as occurs if both the light- and the heavy-atom substructures are centrosymmetric or close to it. On the other hand, if the light-atom substructure is clearly noncentrosymmetric, the heavy-atom substructure may be (pseudo-) centrosymmetric or contain some other (pseudo-) symmetry operation of the second kind. In other words, the inversion-distinguishing power of an $\mathrm{X}$-ray diffraction experiment is increased by the inclusion of dispersive scatterers, there being very few restrictions on the position of the dispersive atoms within the crystal structure. A simple application of this effect is to cocrystallize the compound investigated with a molecule such as $\mathrm{CCl}_{4}$. In such a way, an achiral dispersive molecule may be used in the determination of the absolute configuration of a chiral nondispersive molecule.

\subsection{Intensity data}

It is commonly admitted, albeit reluctantly, that in a satisfactory raw intensity data set there should be at least one measurement from each set of symmetryequivalent reflections out to the resolution limit chosen for the study. From this raw set, a reduced data set of unique reflections is produced by averaging symmetryequivalent reflections. For the purposes of structure refinement and absolute-structure determination of a noncentrosymmetric crystal structure, the correct symmetry to use for the averaging process is that of the crystal class, in which, of course, $\bar{h} \bar{k} \bar{l}$ is not symmetryequivalent to $h \mathrm{kl}$. Use of a symmetry other than that of the crystal class, for example the Laue symmetry, needs to be justified in each particular case. In principle, there is an advantage to keeping $h k l$ and $\bar{h} \bar{k} \bar{l}$ separate, even if the absolute structure of the crystal is known at the outset. To demonstrate this advantage, consider the halfsum, $S$, and the half-difference, $D$, of the squared model structure amplitudes dependent on $x$, the Flack (1983) parameter:

$$
\begin{aligned}
S & =\frac{1}{2}\left[G^{2}(h, k, l, x)+G^{2}(\bar{h}, \bar{k}, \bar{l}, x)\right] \\
& =\frac{1}{2}\left[|F(h, k, l)|^{2}+|F(\bar{h}, \bar{k}, \bar{l})|^{2}\right]
\end{aligned}
$$

and

$$
\begin{aligned}
D & =\frac{1}{2}\left[G^{2}(h, k, l, x)-G^{2}(\bar{h}, \bar{k}, \bar{l}, x)\right] \\
& =\frac{1}{2}(1-2 x)\left[|F(h, k, l)|^{2}-|F(\bar{h}, \bar{k}, \bar{l})|^{2}\right] \\
& =1 / 2(1-2 x) \Delta .
\end{aligned}
$$

$S$ is independent of $x$ and thus contains only structural information corresponding to a $50 \%$ inversion-twin model. $D$ may be systematically zero over a whole set of data, in which case $D$ evidently contains no structural information. This occurs either when $x=\frac{1}{2}$, an equivolume inversion twin, or when $\Delta=0$, a situation studied in the previous paragraph. With $D$ not systematically zero, it is not possible to write $D$ as a function of $x$ and $S$ only. Thus, from the functional form of $D$, one sees that it contains both information on the distinguishability of the structure from its inverse and structural information supplementary to that contained in $S$. The information content of crystal-class averaged data is higher or at least equal to that of Laue-symmetry averaged data. An informal measure of the excess structural information in crystal-class averaged data is afforded by its capacity to phase reflections in single or multiple anomalous-scattering techniques or to define the Flack (1983) parameter during refinement. 


\section{Determination of absolute structure by $X$-ray diffraction using an internal chiral reference}

The presence in a crystal structure of enantiopure chiral molecules, groups or chiral centres of known absolute configuration leads directly to the determination of the absolute structure of the whole crystal by making the image of the atomic arrangement correspond to that of the known chiral molecule. The chiral molecules (or groups or centres) thus act as an internal reference. It may be introduced as part of the crystal by chemical reaction or cocrystallization using an enantiopure sample of the reference substance. The internal-reference technique is necessarily limited to chiral crystal structures due to the enantiopurity of the reference substance. By using an internal chiral reference, the determination of the absolute structure does not need dispersive scatterers to be present. If present, the effects of anomalous scattering must confirm the known absolute configuration of the reference. It is important to stress that the correctness of absolute-structure determination using an internal chiral reference depends crucially on the knowledge of the enantiopurity of the reference material and its indicated absolute configuration.

\section{Determination of absolute configuration from absolute structure}

Bijvoet (1949) and Peerdeman et al. (1951) achieved the first determination of absolute configuration by X-ray diffraction. In the terms used here, we would say that the absolute structure of the crystal was first determined by single-crystal X-ray diffraction with dispersive scatterers followed by the deduction of the absolute configuration from this.

Not all valid determinations of absolute structure can necessarily lead to the assignment of an absolute configuration.

Space-group restriction: the simplest restriction is one of space-group symmetry. If the space group contains symmetry operations of the second kind, it must occur that these operate either intramolecularly, forcing the individual molecules to be achiral, or intermolecularly, forcing an arrangement of pairs of opposite enantiomers. Thus, in the first case, the molecules are achiral and in the second a racemate is present. It is only in crystals displaying space groups containing exclusively symmetry operations of the first kind (point groups: 1, 2, $222,4,422,3,32,6,622,23,432)$ that the determination of absolute configuration is possible.

Chiral molecular entity restriction: to comply with the definition of absolute configuration, one needs to identify a chiral molecular entity and its spatial arrangement in the crystal structure. For example, Bijvoet (1949) and Peerdeman et al. (1951) established the absolute configuration of the tartrate anion (a chiral molecule) but correctly made no claims to have performed so for the sodium or rubidium cations, which are not molecules. The intramolecular symmetry operations of the second kind forcing a molecule to be achiral are either crystallographic (see space-group restriction) or noncrystallographic. The spatial arrangement of a candidate molecule for absolute-configuration determination must be examined for noncrystallographic symmetry operations of the second kind. If any are found, the molecule is achiral and its absolute configuration cannot be determined.

Solid-state enantiopurity restriction: one needs to verify that all occurrences in the crystal structure of the chiral molecular entity are the same enantiomer. When the space group contains no symmetry operations of the second kind (see space-group restriction) and the asymmetric unit contains more than one occurrence of the chiral molecule, the spatial arrangements of these molecules must be examined to see if they are the same enantiomer. All occurrences of a chiral molecule in a crystal structure must have the same chirality sense for an absolute-configuration assignment to be valid.

\section{Characterization of crystals and chiral molecules}

For absolute-structure and absolute-configuration determinations to have a practical value, their results need to be applicable to other crystals or samples obtained from the bulk product. The sample used needs to be physically characterized to permit the absolutestructure or absolute-configuration determination to be reliably applicable to other samples. In absoluteconfiguration determination, the chiral molecules that constitute the bulk substance are the subject of interest and it would seem important to characterize them in the form that they are used by the chemist, most frequently, in solution. The absolute configuration of a molecule as determined by crystal structure analysis is that of the solid state. The spatial arrangement of the atoms in a molecule in the solid state may not be the same as that in solution.

\subsection{Characterization of crystals}

Will every single crystal taken from the same batch or even from different batches have the same absolute structure? For crystals formed by the crystallization of an enantiopure substance, the crystals will be chiral and all of the same enantiomorph. In this short section, on the other hand, we will recall a few important cases in which individual crystals, or the molecules in the crystals, are not characteristic of the bulk substance. This list is not exhaustive. The subject of enantiomers, racemates and resolutions is dealt with in detail by Jacques et al. (1994) and more briefly but more recently by Eliel \& Wilen (1994).

A racemic conglomerate may be formed by spontaneous resolution in the crystallization of a racemate. 
Taking an arbitrary choice of crystal, the enantiomorph will be arbitrary. When the crystallization system contains an excess of one enantiomer, a mixture may be formed containing an excess of the one enantiomorph corresponding to the enantiomer in excess and an arbitrary choice of crystal may not be of that enantiomorph that is in excess.

Crystals with a chiral structure formed from achiral molecules in solution are a mechanical mixture of the two enantiomorphs. This is not a racemic conglomerate since the molecule is achiral. The proportion of the two enantiomorphs in the mechanical mixture is not fixed, and the phase diagram of this system and that of a racemate forming a racemic conglomerate are essentially different. Taking an arbitrary choice of crystal, the enantiomorph will be arbitrary.

In the preparation of crystals containing an internal chiral reference, the starting solution contains either the pure enantiomer $R$ or the pure enantiomer $S$ or perhaps a mixture of the two in unknown proportion. This solution is reacted or cocrystallized with another molecule $R^{\prime}$, known to be enantiomerically pure. $R-R^{\prime}$ and $S-R^{\prime}$ are diastereoisomers and hence will be formed at different rates. The equilibrium point of the two reactions will be different. The solubility of $R-R^{\prime}$ and $S-R^{\prime}$ will be different. Under these conditions, it is easy to see that the proportion of crystals $R-R^{\prime}$ and crystals $S-R^{\prime}$ will not correspond to the proportion of the starting enantiomers $R$ and $S$ in solution. Only in the case of enantiomerically pure starting material will any crystal be characteristic of it.

\subsection{Characterization of chiral molecules}

Classically, optical activity has been used as a fingerprint to characterize chiral molecules, since the symmetry restrictions on optical activity in solution are identical to those of enantiomers (the same is not true for the crystalline state). However, measurement of the optical rotation does not of itself establish the enantiopurity of a substance. Calculation of optical activity is very tricky, even if the molecular structure is known. Moreover, optical activity is dependent on wavelength, concentration and solvent. For the synthetic chemist, measurement of optical activity has taken a secondary role behind modern physicochemical techniques, such as asymmetric chromatography on enantioselective stationary phase, NMR, mass spectroscopy etc. Once calibrated correctly with a suitable mixture of enantiomers, these techniques are capable of establishing the enantiopurity of a substance and even in some cases of separating enantiomers.

\section{Determination of absolute structure in twinned crystals}

A twinned crystal may be viewed as a solid-state agglomerated mixture of rotated and/or inverted copies of the untwinned crystal structure. Each component in this mixture is specified by two attributes.

(i) The volume fraction $x_{i}$ of the $i$ th component in the macroscopic crystal. This value may be established during structure refinement.

(ii) The Euclidean isometry relating the orientation of the component to that of the basic one. This twinsymmetry operation may be established by arguments of symmetry (Janovec, 1972; Flack, 1987) and is not unique. It comes from a group $G$ of Euclidean isometries of which the crystal point group $P$ is a subgroup, $G \supset P$. The twin-symmetry operation is in fact a representative of a left coset in the decomposition of $G$ with respect to $P$.

\subsection{Rotation-only point groups}

We will deal solely with the case in which $P$ is one of the 11 noncentrosymmetric point groups containing only rotations (point groups: 1, 2, 222, 4, 422, 3, 32, 6, 622, 23, 432 ); the analysis is simple and the results are of use in the determination of absolute configuration. The symmetry operations of $P$, being operations of the first kind, have a determinant of +1 in their matrix representation. Thus, in the left coset decomposition of $G$ with respect to $P$, it is evident that all the symmetry operations in any one coset will have the same determinant (either +1 or -1 ) since these are produced by multiplying the symmetry operations of $P$ on the left by one and the same symmetry operation drawn from $G$. It results that for a rotation-only point group, although any twin-symmetry operation is not unique, the determinant of equivalent twin laws is invariant. Twin symmetry operations of determinant +1 will produce a rotatedonly image of the basic structure, whereas those with determinant -1 will produce a rotated and inverted image. For the purposes of the analysis of absolute structure, the total amount of rotated-only structure, $x^{+}$, may be deduced by summing the volume fractions corresponding to twin laws of determinant +1 , $x^{+}=\sum x_{i}^{+}$, and that of rotated-and-inverted structure, $x^{-}$, may be deduced by summing the volume fractions corresponding to twin laws of determinant -1 , $x^{-}=\sum x_{i}^{-} \cdot x^{-}$is the equivalent of the Flack $x$ parameter for multiply twinned crystals. It must be emphasized that the above analysis does not apply to the ten noncentrosymmetric point groups containing symmetry operations of the second kind (point groups: $m, m m 2, \overline{4}$, $\overline{4} 2 m, 4 m m, 3 m, \overline{6}, \overline{6} m 2,6 m m, \overline{4} 3 m)$ nor to the 11 centrosymmetric point groups (point groups: $\overline{1}, 2 / m$, $m m m, 4 / m, 4 / m m m, \overline{3}, \overline{3} m, 6 / m, 6 / m m m, m \overline{3}, m \overline{3} m)$.

\subsection{Example 1 of Herbst-Irmer \& Sheldrick (1998)}

Consider example 1 of Herbst-Irmer \& Sheldrick (1998), a twinned crystal of a compound containing a hydridochlorocarbonyltris(triphenylphosphine)osmium- 
(II) ion in space group $P 3_{1}$. This space group contains only symmetry operations of determinant +1 and the above analysis may be applied. The structure was refined as a four-component twin: ' $k_{2}=0.064$ (13) for matrix $010,100,00-1, \quad k_{3}=0.038(17)$ for matrix $-100,0-10,00-1$ and $k_{4}=0.329(13)$ for matrix $0-10,-100,001$ '. $k_{1}$ may be obtained from the relationship $k_{1}=1-k_{2}-k_{3}-k_{4}$ to give $k_{1}=0.569$ (14) for matrix 100,010,001. The twin symmetry operations are of determinant +1 for matrices 1 and 2 , and -1 for matrices 3 and 4 . In the nomenclature of the current analysis, one has $x_{1}^{+}\left(=k_{1}\right)=0.569(14), x_{2}^{+}\left(=k_{2}\right)=$ $0.064(13), x_{1}^{-} \quad\left(=k_{3}\right)=0.038(17)$ and $x_{2}^{-}\left(=k_{4}\right)=$ 0.329 (13), giving $x^{+}\left(=x_{1}^{+}+x_{2}^{+}\right)=0.633(17)$ and $x^{-}$ $\left(=x_{1}^{-}+x_{2}^{-}\right)=0.367(17)$. The standard uncertainty of $x^{-}$(equivalent to the Flack $x$ parameter for this multiply twinned crystal) is low and one thus sees that the inversion-distinguishing power of the measurements is very good, as expected by Herbst-Irmer \& Sheldrick (1998). The experiment clearly shows that $63 \%$ of the crystalline sample contains the structure determined by Herbst-Irmer \& Sheldrick (1998) in space group $P 3_{1}$ and $37 \%$ contains the inverted structure in space group $P 3_{2}$. From these measurements, it is clearly neither possible to establish the absolute structure nor possible to establish the absolute configuration for this compound, for which in any case the synthesis was probably not stereospecific.

\section{Defining absolute structure and absolute configuration}

The definition of absolute structure as given in the glossary $(\$ 1)$ appears here for the first time in print, whereas that of absolute configuration is essentially the IUPAC definition (Moss, 1996) with a small but most significant modification. The two definitions are written in the same style in order to emphasize their similarities and differences.

Our modification of the IUPAC definition of absolute configuration consists of the adjunction of the words physically identified as a qualifier to chiral molecular entity. It is thought of importance to add these words as there is little use of an absolute-configuration determination if the substance studied cannot be reliably identified at a later stage. On this point, the IUPAC (1996) definition has already been criticized by Eliel (Eliel \& Wilen, 1994) and Collet (1998) and it is instructive to note that previously Jacques et al. (1994) insisted that the optical activity of the substance should be reported for an absolute-configuration determination to be valid. As mentioned above, other techniques are now also available. The expression physically identified has also been included in the definition of absolute structure, echoing the plea of Glazer \& Stadnicka (1989) that, as part of absolute-structure determination, the characterization of the crystal itself by measurement of a physical, chemical or morphological property be undertaken and reported. These authors also provide a scheme for the consistent naming of the property.

The definition of absolute configuration makes it clear that the term is to be applied only to chiral molecular entities and not to whole crystals. This is in agreement with the recommendations of Glazer \& Stadnicka (1989) '...that the term absolute configuration should be reserved only for molecular species and not for crystal structures'. We concur with Glazer \& Stadnicka on this point.

Jones (1998) in his definition (Jones, 1984a) of the expression 'determination of absolute structure', viz "... it is often necessary to consider carefully exactly which manifestation of noncentrosymmetry is being determined by the analysis of anomalous scattering effects. It is unfortunate that there is no general expression corresponding to 'determination of absolute configuration (or conformation) or of polar-axis direction or resolving the ambiguity of enantiomorphic space-group pairs or of axis directions' ... I suggest the use of 'determination of absolute structure' ...", wished to provide a blanket term for the entity now expressed by the Flack (1983) parameter. Several factors make us think that the time is now opportune to make much finer use of absolute structure. Firstly, in the reporting of an analysis of a noncentrosymmetric structure which does not seek to determine absolute structure or absolute configuration, it is sufficient to state the value and standard uncertainty obtained for the Flack parameter as proof that a polar-dispersion error has not been made. In this case, no physical interpretation of the Flack parameter is undertaken and no physical characterization of the crystal or molecules is necessary. Use of 'determination of absolute structure' according to Jones may more clearly be expressed by 'refinement of the Flack (1983) parameter'. Secondly, for a property to be worthy of carrying the absolute qualifier, it must be adequately physically identified. Thirdly and lastly, it is natural for a crystal that a property structure should describe a spatial arrangement of atoms represented by a list of atomic coordinates. As defined, absolute structure takes on a clear and useful meaning, ensuring its continued use into the future.

\section{Concluding remarks}

On ne peut être trop prudent dans les conclusions à
déduire de l'expérience, lorsque l'on a affaire à des
substances quelquefois si semblables en apparence, et
qui peuvent être au fond si différentes. (Pasteur, 1848.)

In order to clarify the comprehension and presentation of absolute structure, we have separated different but interacting concepts under different names. Absolute structure describes the spatial arrangement of atoms in a noncentrosymmetric crystal structure for 
which the structure has been clearly distinguished from its inverse in a point. This is in contrast to an ambiguous structure determination where the results of the analysis are a choice between the two centrosymmetrically related images. The inversion-distinguishing power of a diffraction experiment measures its capacity to distinguish between a crystal structure and its inverse. An experiment with high inversion-distinguishing power has the capacity to lead to an absolute-structure determination, whereas a low inversion-distinguishing power permits only an ambiguous structure determination. Inversion-distinguishing power may be increased in an X-ray diffraction experiment either by the inclusion of dispersive scatterers in the crystal structure, there being very few restrictions on their position within the molecule or crystal structure, or by suitably tuning the wavelength. A measure of the inversion-distinguishing power of a diffraction experiment is afforded by the reciprocal of the standard uncertainty of the Flack (1983) parameter.

The underlying difficulty of the treatment of mixtures has appeared at different points in this text. In contrast to pure, and in particular enantiopure, substances, the crystals of which contain only molecules of one single kind, impure substances, and in particular racemates and samples with an enantiomeric excess, show a wide diversity in their modes of crystallization and reactivity. It cannot be stressed sufficiently that small amounts of impurity, and in particular the opposite enantiomer as impurity in an 'enantiopure' substance, may well be the source of otherwise inexplicable results. Inversion twins and multiply twinned crystals have been touched upon. These also are mixtures of a special kind requiring careful treatment.

Caution is always required in transferring an absolute-configuration assignment to the bulk substance knowing that it has been derived from measurements on only one single crystal and is characteristic of the molecule in the solid state. It would seem a meagre precaution for the publication of absolute-configuration determinations that proof of the enantiopurity of the bulk sample should be provided. Furthermore, measurement of the optical activity in solution continues to provide a useful fingerprint and control. On the other hand, for those structure determinations of which the primary objective is in the field of structure-property relations, it is reasonable to expect that absolute-structure determination will be accompanied by the measurement of some chemical, physical or morphological property of the crystal.

The authors wish to thank A. J. Blake, W. Clegg, A. Collet, J. D. Dunitz, A. M. Glazer, Y. Grin, S. R. Hall, P. G. Jones, J. Lacour, A. Linden, D. Schwarzenbach,
A. L. Spek, D. J. Watkin and the two referees for their invaluable comments on this paper.

\section{References}

Bernardinelli, G. \& Flack, H. D. (1985). Acta Cryst. A41, 500-511.

Bernardinelli, G. \& Flack, H. D. (1987). Acta Cryst. A43, 75-78.

Bijvoet, J. M. (1949). Proc. K. Ned. Akad. Wet. Ser. B, 52, 313-314.

Collet, A. (1998). Private communication.

Coster, D. S., Knol, K. S. \& Prins, J. A. (1930). Z. Phys. 63, 345-369.

Cruickshank, D. W. J. \& McDonald, W. S. (1967). Acta Cryst. 23, 9-11.

Eliel, E. L. \& Wilen, S. H. (1994). Stereochemistry of Organic Compounds. New York: John Wiley.

Flack, H. D. (1983). Acta Cryst. A39, 879-881.

Flack, H. D. (1987). Acta Cryst. A43, 564-568.

Flack, H. D. \& Bernardinelli, G. (1999). Acta Cryst. In preparation.

Flack, H. D. \& Schwarzenbach, D. (1988). Acta Cryst. A44, 499-506.

Glazer, A. M. \& Stadnicka, K. (1989). Acta Cryst. A45, 234-238.

Hall, S. R., Allen, F. H. \& Brown, I. D. (1991). Acta Cryst. A47, 655-685.

Hamilton, W. C. (1965). Acta Cryst. 18, 502-510.

Herbst-Irmer, R. \& Sheldrick, G. M. (1998). Acta Cryst. B54, 443-449.

Jacques, J., Collet, A. \& Wilen, S. H. (1994). Enantiomers, Racemates and Resolutions. Reissue with corrections. Malabar, FL: Krieger.

Janovec, V. (1972). Czech. J. Phys. B, 22, 974-994.

Jones, P. G. (1984a). Acta Cryst. A40, 660-662.

Jones, P. G. (1984b). Acta Cryst. A40, 663-668.

Jones, P. G. (1986a). Acta Cryst. A42, 57.

Jones, P. G. (1986b). Acta Cryst. A42, 942.

Jones, P. G. (1998). Private communication

Jones, P. G. \& Meyer-Bäse, K. (1987). Acta Cryst. A43, 79-80.

Jones, P. G., Schelbach, R., Schwarzmann, E. \& Thöne, C. (1988). Acta Cryst. C44, 1196-1198.

Moss, G. P. (1996). Pure Appl. Chem. 68, 2193-2222. [Basic Terminology of Stereochemistry, IUPAC Recommendations 1996, available at http://www.Chem.qmw.ac.uk/iupac/ stereo/.]

Pasteur, L. (1848). Ann. Chim. Phys. 24, 442-459.

Peerdeman, A. F., van Bommel, A. J. \& Bijvoet, J. M. (1951). Proc. K. Ned. Akad. Wet. Ser. B, 54, 16-19.

Rogers, D. (1975). Anomalous Scattering, edited by S. Ramaseshan \& S. C. Abrahams, pp. 231-250. Copenhagen: Munksgaard.

Rogers, D. (1981). Acta Cryst. A37, 734-741.

Sheldrick, G. M. (1993/1997). SHELXL93/SHELXL97. Program for the Refinement of Crystal Structures. University of Göttingen, Germany.

Ueki, T., Zalkin, A. \& Templeton, D. H. (1966). Acta Cryst. 20, 836-841. 